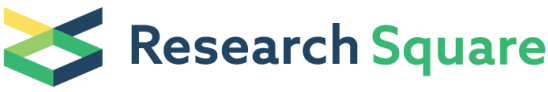 \\ Preprints are preliminary reports that have not undergone peer review. They should not be considered conclusive, used to inform clinical practice, or referenced by the media as validated information.
}

\section{Characterization of TGF $\beta$-Associated Molecular Features and Drug Responses in Gastrointestinal Adenocarcinoma}

\section{Qiaofeng Zhang}

Hepatic Surgery Center, Tongji Hospital, Tongji Medical College, Huazhong University of Science and Technology, Wuhan, Hubei 430030, China.

\section{Furong Liu}

Hepatic Surgery Center, Tongji Hospital, Tongji Medical College, Huazhong University of Science and Technology, Wuhan, Hubei 430030, China.

\section{Lu Qin}

Department of Anesthesiology, Union Hospital, Tongji Medical College, Huazhong University of Science and Technology, Wuhan 430022, China.

\section{Zhibin Liao}

Hepatic Surgery Center, Tongji Hospital, Tongji Medical College, Huazhong University of Science and Technology, Wuhan, Hubei 430030, China.

\section{Jia Song}

Hepatic Surgery Center, Tongji Hospital, Tongji Medical College, Huazhong University of Science and Technology, Wuhan, Hubei 430030, China.

\section{Huifang Liang}

Hepatic Surgery Center, Tongji Hospital, Tongji Medical College, Huazhong University of Science and Technology, Wuhan, Hubei 430030, China.

\section{Xiaoping Chen}

Hepatic Surgery Center, Tongji Hospital, Tongji Medical College, Huazhong University of Science and Technology, Wuhan, Hubei 430030, China.

\section{Zhanguo Zhang}

Hepatic Surgery Center, Tongji Hospital, Tongji Medical College, Huazhong University of Science and Technology, Wuhan, Hubei 430030, China.

\section{Bixiang Zhang ( $\sim$ bixiangzhang@163.com )}

Hepatic Surgery Center, Tongji Hospital, Tongji Medical College, Huazhong University of Science and Technology, Wuhan, Hubei 430030, China.

\section{Research Article}


Keywords: Gastrointestinal adenocarcinoma, transforming growth factor beta, multi-omics signatures, drug susceptibility, deep neural network

Posted Date: January 22nd, 2021

DOI: https://doi.org/10.21203/rs.3.rs-144904/v1

License: (1) This work is licensed under a Creative Commons Attribution 4.0 International License. Read Full License

Version of Record: A version of this preprint was published at BMC Gastroenterology on July 12th, 2021. See the published version at https://doi.org/10.1186/s12876-021-01869-4. 
1 Characterization of TGF $\beta$-associated molecular features and drug

3 Qiaofeng Zhang1,2,3†, Furong Liu ${ }^{1,2,3 \dagger}$, Lu Qin ${ }^{4}$, Zhibin Liao ${ }^{1,2,3}$, Jia Song ${ }^{1,2,3}$,

4 Huifang Liang ${ }^{1,2,3}$, Xiaoping Chen ${ }^{1,2,3}$, Zhanguo Zhang ${ }^{1,2,3 *}$, Bixiang Zhang 1,2,3*

5 1. Hepatic Surgery Center, Tongji Hospital, Tongji Medical College, Huazhong 6 University of Science and Technology, Wuhan, Hubei 430030, China.

2. Hubei Province for the Clinical Medicine Research Center of Hepatic Surgery, Wuhan, Hubei 430030, China.

3. Hubei key laboratory of Hepato-Pancreato-Biliary Diseases, Tongji Hospital, Tongji Medical College, Huazhong University of Science and Technology, Wuhan, Hubei 430030, China.

4. Department of Anesthesiology, Union Hospital, Tongji Medical College, Huazhong University of Science and Technology, Wuhan 430022, China.

$\uparrow$ Qiaofeng Zhang and Furong Liu contributed equally to this work.

\section{Corresponding Authors}

Prof. Zhanguo Zhang, or Bixiang Zhang

Hepatic Surgery Center, Tongji Hospital, Tongji Medical College, Huazhong University of Science and Technology, Hubei Province for the Clinical Medicine Research Center of Hepatic Surgery, 1095 Jiefang Avenue, 430030 Wuhan, China. Tel.: +86 27 83663400; fax: +86 27 83662851; E-mail: zhanguo_tjh@hust.edu.cn, bixiangzhang@,163.com 
Background: Gastrointestinal adenocarcinoma (GIAD) has caused a serious disease burden globally. Targeted therapy for the transforming growth factor beta (TGF- $\beta$ ) signaling pathway is becoming a reality. However, the molecular characterization of TGF- $\beta$ in GIAD requires further exploration.

Results: The TGF- $\beta^{\text {high }}$ group had a worse prognosis in overall GIAD patients, and had a worse prognosis trend in gastric cancer and colon cancer specifically. Signatures (including mRNA and proteins) of the TGF- $\beta^{\text {high }}$ group is highly correlated with EMT. According to miRNA analysis, miR-215-3p, miR-378a-5p, and miR-194-3p may block the effect of TGF- $\beta$. Further genomic analysis showed that TGF- $\beta^{\text {low }}$ group had more genomic changes in gastric cancer, such as TP53 mutation, EGFR amplification, and SMAD4 deletion. And drug response dataset revealed sensitive drugs or drug resistant drugs corresponding to TGF- $\beta$ associated mRNAs. Finally, the DNN model showed an excellent predictive effect in predicting TGF- $\beta$ status in different GIAD datasets.

Conclusions: Our study provided a comprehensive analysis of the molecular characteristics associated with TGF- $\beta$ and provides possible therapeutic targets in GIAD.

Keywords: Gastrointestinal adenocarcinoma, transforming growth factor beta, multiomics signatures, drug susceptibility, deep neural network 


\section{Introduction}

Cancer of the digestive tract share a large quantity of global cancer incidences. gastrointestinal adenocarcinomas (GIADs), including esophageal adenocarcinoma (ESAD), stomach adenocarcinoma (STAD), colon adenocarcinoma (COAD), rectum adenocarcinoma (READ), revealed a nonnegligible global health burden in recent years [1]. Previous studies have established that GIADs share the same features of DNA hypermethylation, mRNA expression and protein biomarkers, which confirmed GIAD in possession of exclusive characteristics [2, 3]. Apart from traditional histologic classification, an increasing number of molecular signatures were found across cancer. Models and clinical cohort were built to identify the function of molecular biomarkers. Thus, a dramatic increasing number of potential biomarkers were found and reported, which made clinical diagnosis, prognostic and immunotherapy more effective and reliable.

Transforming growth factor beta (TGF- $\beta$ ) family consists great many of activation and inhibition factors which participate in a variety of cellular biological processes [4]. As a prototypical factor in TGF- $\beta$ family proteins, encoded by 33 genes in mammals, TGF$\beta$ is a multifunctional regulator involved in cell proliferation and differentiation [5], even in immune suppression within tumor microenvironment [6]. Some cell-surface transmembrane receptors with serine or threonine kinase activity can interact with activated TGF- $\beta$ s, following phosphorylation of SMAD proteins, which then regulate the expression of TGF- $\beta$ target genes [7]. Additionally, the activation of TGF- $\beta$ signaling pathways can be negatively correlated with the development of COAD in multiple mechanisms [8].And target genes of TGF- $\beta$ are upregulated in ESAD samples while tumor progression is suppressed by TGF- $\beta$ knockdown [9].

With the TGF- $\beta$ signal transduction cascades being able to promote the growth and differentiation of tumor cells, and to inhibit cell proliferation in different tumor stages $[4,7,10]$, it is still difficult to define the function of TGF- $\beta$ signaling pathways in digestive tract adenocarcinomas. Moreover, TGF- $\beta$ signaling targeted drugs have bright prospect in clinical application. Hence, more prospective research for TGF- $\beta$ associated 
molecular signatures and possibility of targeted therapy in GIADs are in need.

We focused on multi-omics study across 4 cancer types in The Cancer Genome Atlas (TCGA) to elaborate different molecular pattern between TGF- $\beta$ high expression (TGF$\beta^{\text {high }}$ ) and low expression (TGF- $\beta^{\text {low }}$ ) cluster. An array of transcription, postmodification, proteomic change, gene mutation, genomic alteration and what regulating function they induced were analyzed in cancers. And the potential effects of anticancer drugs targeted on TGF- $\beta$ signaling were assessed.

\section{Methods and Materials}

\section{TCGA and GEO data collection and processing}

We collected multi-omics data (including count data of RNA-seq and miRNA-seq, Reverse phase protein lysate microarray (RPPA), mutation maf files and survival data) of 4 type of gastrointestinal adenocarcinoma (ESAD, STAD, COAD, READ) from Xena Hub. Genes with average expression of less than 1 in RNA-seq and miRNA-seq as well as synonymous mutations in the mutation data were filtered. Level 4 Copy number variation (CNV) data processed by GISTIC2.0 was obtained from Firehouse. Additional sequencing data for gastrointestinal adenocarcinoma were downloaded from NCBI GEO, including GSE19417, GSE62254, GSE17536, GSE45404, along with GSE62254 and GSE17536 contained survival data.

\section{Classification of TGF- $\beta$ status in GIAD}

39 core TGF- $\beta$ genes were selected as TGF- $\beta$ gene expression signatures, including TGF- $\beta$ ligands, receptors, receptor substrates, Co-SMAD, inhibitory Smads, adaptors, and Smad chaperones. In order to evaluate different TGF- $\beta$ levels, two evaluation methods were used: 1. Unsupervised K-means clustering analysis based on the 39 mRNA of TGF- $\beta$-related signatures for each sample in GIAD; 2 . Gene set variation analysis based on single sample gene-set enrichment analysis (ssGSEA) method was used to calculate the TGF- $\beta$ score of each sample in GIAD. Unsupervised clustering 
was performed by R package 'ConsensuClusterPlus' [11], which is repeated 1000 times to ensure the stability of the results as in previous report [12].

\section{Analysis of alterations between different TGF- $\beta$ status}

For the mRNA, miRNA, and RPPA data in TGF- $\beta^{\text {high }}$ and TGF- $\beta^{\text {low }}$ group in each GIAD, we used the permutation test for differential analysis as previously described [13]. For RNA-seq, we further screened mRNAs with the absolute value of $\log \mathrm{FC}\left(\log _{2}\right.$ (Fold change $)=$ mean value in TGF- $\beta^{\text {high }} /$ mean value in TGF- $\left.\beta^{\text {low }}\right)>1$; for miRNA, we set the absolute value of $\log \mathrm{FC}>0.5$ as the threshold; for RPPA data, we set the difference greater than 0.2 as the significantly changed proteins. For the mutation data, we selected high frequency mutations of more than $10 \%$ in the TGF- $\beta^{\text {high }}$ or TGF- $\beta^{\text {low }}$ group for analysis. Also, for $\mathrm{CNV}$ data, amplification or deletion regions exceeding $10 \%$ in the TGF- $\beta^{\text {high }}$ or TGF- $\beta^{\text {low }}$ group were selected for analysis. Fisher's exact test was used to evaluate significantly mutated genes, amplified and deleted chromosomal regions. We used FDR correction for all multiple tests, with FDR $<0.05$ set as the threshold. Additionally, miRNA-targeted mRNAs were extracted from miRTarBase [14] and miRDB [15]. The mRNAs targeted by miRNA in two databases was intersected as the final targeted mRNAs. In order to analyze the correlation between miRNA and mRNA in different TGF- $\beta$ status groups, principal component analysis (PCA) was used to reduce dimensionality to obtain the first principal component (PC1), and then Pearson correlation evaluation was conducted for the PC1. The advantage of this approach is that it concentrates on scores that are correlated (or inversely correlated) between gene sets.

\section{Enrichment analysis of pathways and gene set functions}

We respectively performed Kyoto Encyclopedia of Genes and Genomes (KEGG) pathway analysis of the differentially expressed mRNAs in each type of GIAD, including genes upregulated in the TGF- $\beta^{\text {high }}$ or TGF- $\beta^{\text {low }}$ group. At the same time, geneset enrichment analysis (GSEA) analysis was also used to analyze the significant up- 
or down-regulated pathways and functions in TGF- $\beta^{\text {high }}$ group involved in each GIAD. Both KEGG and GSEA analyses are implemented on the R package 'clusterProfiler' [16]. The classic 'Hallmark gene sets' was selected as signatures for GSEA analysis. FDR $<0.05$ was considered as significant enrichment.

\section{Analysis of drug-targeted TGF- $\beta$ associated with gene signatures}

The Pearson coefficient $(r)$ between the expression of TGF- $\beta$ specific mRNAs and the drug response (IC50) were evaluated in digestive tract cell lines. The drug response data of cell lines was downloaded from Genomics of Drug Sensitivity in Cancer (GDSC) [17]. Pearson correlation coefficient and FDR were calculated in GDSC, in which $|r|>$ 0.3 and FDR $<0.05$ were considered to be significant. We also further evaluated the estimated drug responses of 138 drugs in the TCGA sample from a previous study [18]. For these estimated drug responses, we used the Wilcoxon signed rank test, and FDR $<$ 0.05 was considered to be differential.

\section{Construction of deep neural network (DNN)}

Using genomic data, we trained a deep neural network (DNN) model to predict the TGF- $\beta$ status of different GIAD datasets (Fig. 7a). In addition to the input layer, we construct a four-layer neural network, including three hidden layers and an output layer. We labeled the TGF- $\beta^{\text {high }}$ group as 1 and the TGF- $\beta^{\text {low }}$ group as 0 . For a given hidden layer $l$, we use ReLu activation function. According to the output layer of the previous layer as the input of the next layer $A^{[l-1]}$, we can carry out forward propagation as follows:

$$
A^{[l]}=\max \left(W^{[l]} A^{[l-1]}+b^{[l]}, 0\right),\left(A^{[0]}=X, l=(1,2,3)\right)
$$

Where $A^{[l]}$ is the output matrix of the hidden layer, $W^{[l]}$ is the weight matrix, $b^{[l]}$ is a column vector, and $X$ is the normalized matrix of input layer including the samples and gene signatures. Also, for the final output layer, we used the sigmoid activation function, 


$$
Y=\frac{1}{e^{A^{[3]}}+1}
$$

154

Where $\mathrm{Y}$ is the probability vector of the output layer, and $A^{[3]}$ is the output matrix of the third hidden layer. We chose the loss function in logistic regression to measure the operation of the algorithm:

$$
J(W, b)=\frac{1}{m} \sum_{i=1}^{m} L\left(\hat{y}^{(i)}, y^{(i)}\right)=\frac{1}{m} \sum_{i=1}^{m}\left(-y^{(i)} \log \hat{y}^{(i)}-\left(1-y^{(i)}\right) \log \left(1-\hat{y}^{(i)}\right)\right)
$$

Where $J(W, b)$ is the cost function, $\mathrm{m}$ is the sample number, $L\left(\hat{y}^{(i)}, y^{(i)}\right)$ is the lost function of a sample, and $\hat{y}^{(i)}$ and $y^{(i)}$ are output value of DNN and the real label value of sample $i$ respectively. Then, in order to minimize $(W, b)$, we used the stochastic gradient descent training model to learn $W^{[l]}$ and $b^{[l]}$. Learning rate $(\alpha)$ was set to be between 0.0005 and 0.005 , and the epoch was set to be 100 to evaluate the best model. The RMSprop algorithm was used to optimize the model. TCGA data were randomly divided into training set and testing set according to $3: 1.75 \%$ of the TCGA samples were used for training, and the remaining $25 \%$ were used to test the performance of the model, which was evaluated by receiver operating characteristic (ROC) curve. Finally, we used this model to evaluate and analyze the TGF- $\beta$ status on multiple GEO datasets.

\section{Other information for analysis}

All analysis was done on R (Version: 3.6.1) or Python (Version: 3.6). Due to different types of survival data, survival analysis in TCGA refers to overall survival (OS) and progression-free interval (PFI), and in GEO datasets to OS and disease-free survival (DFS). In addition to the statistical analysis mentioned above, the log-rank test was used for survival analysis, and all multiple statistical tests were FDR corrected. 


\section{Result}

\section{Unsupervised classification of TGF- $\beta$ status and overall patterns of multi-omics} signatures between TGF- $\beta^{\text {high }}$ and TGF- $\beta^{\text {low }}$ groups

Unsupervised clustering was used to identify the activation of respective driver genes between two clusters characterized by low and high distribution of TGF- $\beta$ core genes (Fig. 1a). We used single-sample gene set enrichment analysis (ssGSEA) to further exhibit that cluster 2 had higher TGF- $\beta$ scores not only in various categories of GIAD but also in general (Fig. 1b). Correlation analysis was performed to exhibit that TGF- $\beta$ core genes were correlated to TGF- $\beta$ score in a positive tendency (Supplementary Fig. S1a), which explained the consistency of gene activation and cluster outcome. Therefore, we set cluster1 as the TGF- $\beta^{\text {low }}$ group and cluster 2 as the TGF- $\beta^{\text {high }}$ group. To analyze the prognosis of different TGF- $\beta$ groups, we performed a survival analysis. As a whole, GIAD patients with high activation of TGF- $\beta$ pathway had statistically significant poor prognosis $(\mathrm{OS}: \mathrm{HR}=1.374,95 \% \mathrm{CI}=1.095-1.723, \mathrm{P}=0.0058$; PFI: $\mathrm{HR}=1.329,95 \% \mathrm{CI}=1.065-1.659, \mathrm{P}=0.012)($ Fig. $1 \mathrm{c}-\mathrm{d})$. Specific to each tumor, although only STAD showed a significant difference between two groups (Fig. 1e-f), a trend of poor prognosis still shown in TGF- $\beta^{\text {high }}$-specific COAD and ESAD patients (Fig. 1g-h, Supplementary Fig. S1b-e), which partially elucidated the causality of poor prognosis and TGF- $\beta$ pathway activity.

To further investigate TGF- $\beta$ associated molecular features across GIAD, a procedure was designed with analytic pipeline outlined in Fig. 2a. Then, we analyzed various molecular signatures between previous cluster groups, including mRNA expression (approximately 18000 genes), miRNA expression (approximately 600 miRNAs), protein expression (198 proteins), somatic mutation (frequency $>5 \%$ in at least one cluster) and copy number variation (CNV). And the most striking signatures turned out to be mRNA, miRNA and protein expression (Fig. 2b). The number of mRNA genes ranged from 1865 in STAD to 5606 in READ, most of which surprisingly belonged to high score group. The number of miRNAs showing significant expression level ranged 
from 47 in READ to 276 in STAD. Alterations of protein expression exhibited the largest number in STAD, and significant alterations of low score group were more than high score group across cancer types. We also found that STAD had a large amount of alterations in somatic mutation and $\mathrm{CNV}$, while both clusters of other cancer types showed non-differential signatures. In aggregate, STAD got the most TGF- $\beta$ associated signatures, including 1865 mRNA genes, 276 miRNAs, 46 proteins, 18 gene mutation and nearly $90 \mathrm{CNV}$ regions.

\section{TGF- $\beta$ effects on mRNA and functional pathways}

To understand the function of TGF- $\beta$ associated mRNA signatures in digestive tract adenocarcinoma, we compared mRNA expression levels for each gene. Then a robust rank aggregation (RRA) analysis was performed to rank top 50 genes with the highest expression in TGF- $\beta^{\text {high }}$ group as previous research did [19] (Fig. 3a). For example, thrombospondin 4 [20] (THBS4), gremlin 1 [21] (GREM1), slit guidance ligand 2 [22] (SLIT2) and hemicentin-1 [23] (HMCN1, also known as fibulin-6) was able to mediate the function of cell proliferation, invasion and migration induced by TGF- $\beta 1$ (Fig. 3a). Enriched KEGG pathway of differentially expressed mRNAs revealed that genes involved in extracellular matrix signaling, cell adhesion, classic PI3K/AKT signaling pathway and proteoglycans expression were up-regulated in high TGF- $\beta$ score samples (Fig. 3b). Meanwhile, due to the small number of down-regulated genes in the TGF$\beta^{\text {high }}$ group across cancer types, there were fewer common genes and enriched pathways in the four tumors, with the result of pathway enrichment focused on ESAD and STAD (Supplementary Fig. S2a-b). To further manifest the enriched functions of differentially expressed mRNAs, we performed gene set enrichment analysis (GSEA) on all of them (Fig. 3c). Some of the enriched functions across cancer types were associated with TGF- $\beta$ induced biological process, such as epithelial mesenchymal transition (EMT) and inflammatory response, which was involved in tumor genesis and metastasis [6, 24]. We also noticed that signaling pathways of immune regulation and cellular 
metabolism were significantly enhanced. In general, the results were unsurprisingly consistent with previous findings. It is noteworthy that the EMT showed the most significant enrichment in our analysis, suggesting that TGF- $\beta$ signaling pathway in GIAD may promote the occurrence of EMT and tumor metastasis, which is also consistent with previous research reports [25].

\section{Comparison of miRNA, and protein expression in different TGF- $\beta$ status}

As we all known that miRNA is the key regulator in post-transcription of gene expression [12]. In our study, differentially expressed miRNAs were detected across cancer types. Principal component analysis and correlation analysis were implemented to describe the correlation in between TGF- $\beta$ specific expressing miRNAs and their predicted downstream regulation targets, mRNAs. And the results testified a negative regulation function of TGF- $\beta^{\text {low }}$-specific miRNAs by showing a significant negative correlation for 4 types of cancers (Fig. 4a). Meanwhile, the same trend was also found in TGF- $\beta^{\text {high }}$ specifically expressed miRNAs and its targeted TGF- $\beta^{\text {low }}$-specific mRNAs (Supplementary Fig. S3a). These indicated that TGF- $\beta$ associated mRNAs are regulated by the associated TGF- $\beta$-specific miRNAs.

Subsequently, we arranged the top 20 reduced miRNAs, with 3 (miR-215-3p, miR378a-5p, and miR-194-3p) of them significantly downregulated in TGF- $\beta^{\text {high }}$ cluster across cancer type (Fig. 4b). Specifically, miR-215-3p was reported to be a co-inhibitor of cell migration induced by TGF- $\beta 1$ [26]. And the overexpression of miR-194-3p significantly inhibited RUNX2 [27] and PRC1 [28] signaling pathway which was crucial to cell proliferation and migration. Nevertheless, the effects of miR-378a-5p on cell proliferation, migration and invasion were controversial according to recent studies $[29,30]$. The network of these representative miRNAs and enriched target mRNAs were exhibited in Fig. 4c. The expression level of miR-215-3p, miR-194-3p and miR$378 \mathrm{a}-5 \mathrm{p}$ were negatively correlated with TGF- $\beta$ pathway scores across cancer types, which substantiated performed results (Fig. 4d, Supplementary Fig. S3c-d). Though the 
regulatory relationships were not clear between TGF- $\beta^{\text {high }}$-specific expressing miRNAs and corresponding mRNAs, which might owe to few samples of TGF- $\beta^{\text {low }}$-specific mRNAs (Supplementary Fig. S3a-b). A recent research suggested that expression of miR-100 and miR-125b, which got involved in EMT, tumorigenesis and poor prognosis, was induced and upregulated by TGF- $\beta$ [31].

Standardized differences of protein expression levels between two groups were displayed in Fig. 4e. And we focused on STAD, COAD and READ due to unapparent alterations in ESAD samples. For example, downregulated E-cadherin in TGF- $\beta^{\text {high }}$ group suggested its inhibiting effect of metastasis in cancer, which was concordant with previous studies [32, 33]. On the contrary, collagen IV [34] and fibronectin [35] were reported as pro-metastasis factors and found unsurprisingly overexpressed in TGF- $\beta^{\text {high }}$ group. We also found that proteins associated with cell cycle (such as cyclinb1 and cycline1) were downregulated in TGF- $\beta^{\text {high }}$ cluster, which exactly verified the inhibitory effect of TGF- $\beta$ on proliferation [4].

\section{Significant mutations and CNVs between the TGF- $\beta$ subgroups in GIAD}

Various patterns of TGF- $\beta$ signaling were historically found to be associated with genomic stability and gene expression in gastroenteric tumor [36]. Therefore, we analyzed the differences between low and high TGF- $\beta$ expression at the genomic level. Overall, poor genomic stability was found in TGF- $\beta^{\text {low }}$ cluster on account of a higher level of tumor mutation burden (TMB) and CNV, with STAD the most significant one across cancer types (Fig. 5a-b).

Subsequently, we focused on significant gene mutations in individual tumors, and only in COAD and STAD did we find significant mutations (FDR $<0.05$ ). In addition to more EP300 mutation in the TGF- $\beta^{\text {high }}$ group in COAD, we found more interesting results in STAD. Specifically, there were a total of 18 mutated genes with significant difference in 2 STAD subtypes, all of which had more high-frequency mutations in the TGF- $\beta^{\text {low }}$ group (Fig. 5c). As an outstanding tumor suppressor gene activated by 
oncogenic stress, TP53 mutation occurred frequently in various tumors [37], and showed the highest frequency of $52.6 \%$ in our study. Recent studies identified a high correlation between TP53 mutation and tumor immune response [38] with relevant TGF- $\beta$ signaling induced immune evasion and immunotherapeutic resistance [39]. Significant CNVs of STAD in TGF- $\beta^{\text {low }}$ cluster showed that amplification and deletion of genes were concentrated on several chromosomes (Fig. 5d). In particular, EGFR from region $7 \mathrm{p} 11.2$ amplified more frequently in TGF- $\beta^{\text {low }}$ samples $(56.0 \%)$ leading to an increased sensitivity to targeted therapy [40, 41]. Whereas SMAD4 from 18q21.2, as a key mediator of TGF- $\beta$ signaling pathway, exhibited a significant deletion (52.6\%).

\section{Analysis of TGF- $\beta$ associated gene signatures on drug susceptibility in GIAD}

We wanted to further investigate the TGF- $\beta$ signaling specific sensitivity of targeted drugs in digestive tract adenocarcinoma. By identifying a total of 760 differentially expressed mRNA genes associated with TGF- $\beta$, we used Pearson correlation test between gene expression and 50\% inhibiting concentration (IC50) for 345 anticancer drugs to assess drug response across 106 digestive tract cancer cell lines from the Genomics of Drug Sensitivity in Cancer (GDSC). We focused on these significant correlations between 311 genes and 73 drugs, targeting on ERK, JNK and p38 signaling, RTK signaling, chromatin signature, cell cycle, apoptosis and PI3K/mTOR signaling (FDR $<0.05$, Fig. 6a and Supplementary Fig. S4a-h). And dabrafenib got the largest quantity of associated genes (62 genes), such as FAP, FN1 and MMP2 ( $\mathrm{r}_{\mathrm{FAP}}=-0.51, \mathrm{r}_{\mathrm{FN} 1}$ $=-0.42$, rMMP2 $=-0.51)$, which promoted cell invasion, metastatic and EMT [42-44]. Then we calculated imputed tumor response as quantified sensitivity of 138 anticancer drugs in 79 TCGA patient samples (mainly ESAD) to assess the association between drug response and activation level of TGF- $\beta$ signaling pathway. Significant imputed response differences of anticancer drugs were identified with 4 of them resistant but 5 of them sensitive to digestive tract adenocarcinoma (Fig. 6b). In our study, correlations between TGF- $\beta$ score and drug sensitivity that were most significant were exclusively 
analyzed with a positive result for lenalidomide $(r=-0.51)$ but quite opposite for IPA$3(r=0.40)$ (Fig. 6c).

\section{Identification of two TGF- $\beta$ subtypes based on the deep neural network (DNN) associated with TGF- $\beta$ signatures}

In order to better examine the application of TGF- $\beta$ associated signatures in the classification of TGF- $\beta$ subtypes, we developed a DNN model to identify TGF- $\beta$ subtypes. The advantage of DNN is that it can extract the characteristics of the data as much as possible and classify the nonlinear data. We used the top 10 gene signatures as input data. Through the GIAD data marked in TCGA, we trained in $75 \%$ of the data and tested in the remaining data. We found that in TCGA, our model was able to distinguish between the groups with TGF- $\beta^{\text {high }}$ and TGF- $\beta^{\text {low }}$ status. Overall, the DNN classifiers demonstrated excellent performance, with the area under roc curve (AUC) of receiver operating characteristic (ROC) curve across all tumors above $86 \%$ in both the training set and the test set. For example, in STAD, AUC was $96.8 \%$ and $94.6 \%$ in training and test sets, respectively (Fig. 7b).

Further, we analyzed other GIAD datasets. In the other datasets of four types of GIAD, we used the DNN model to identify TGF- $\beta$ subtypes. Obviously, TGF- $\beta$ scores were higher in the DNN-identified high TGF- $\beta^{\text {high }}$ group (Fig. 7c). Meanwhile, we evaluated prognosis in two of the datasets (GSE17536 (COAD) and GSE62254 (STAD)) with survival data. In both datasets, the TGF- $\beta^{\text {high }}$ group had a poorer prognosis, including OS and DFS (Fig. 7d-g), although there was no statistical difference in OS for GSE17536 ( $\mathrm{P}=0.066)$. Finally, we performed GSEA analysis for all TGF- $\beta$ subgroups identified by DNN model in four types of GIAD, in which EMT and TGF- $\beta$ signaling pathways were significantly enriched (all $\mathrm{P}<0.05$, Fig. 7h, Supplementary Fig. S5). These results indicate that our DNN model is a good predictor of TGF- $\beta$ status and patient prognosis in COAD and STAD. 
As a large and classic family of molecules, TGF- $\beta$ s induces a series biological processes involved in tumorigenesis and metastasis, which can be sensitive to targeted drug therapy. The main function of TGF- $\beta$ signaling pathway varies from different cancer types and different stages of cancer. First of all, we performed an unsupervised clustering to characterized expression status of TGF- $\beta$ signaling and associated molecular differences in each cancer type. By identifying the relevancy between TGF$\beta$ high expression and poor prognosis, our method used permutation test to implement multi-omics variation analysis. In this way, we managed to comprehensively evaluate the functions of TGF- $\beta$ signaling by analyzing differentially expressed miRNAs and its negatively regulated mRNAs. In the meantime, identified gene mutations and CNVs demonstrated genetic approaches to a wide range of biological processes, such as cell that TGF- $\beta$ associated gene and protein expression get involved in cancer progression (metastasis in especial), we then used the method from a previous study to identify drug response targeted at aforesaid signatures for further research of targeted therapy [13]. Several drugs suggested a great sensitivity to TGF- $\beta$ induced biological processes of tumor cells. Moreover, some classic oncogenes were found to be targeted by dozens of anticancer drugs, which improved the feasibility of targeted therapy. Also, based on TGF- $\beta$-specific transcriptional signatures, we developed a deep neural network classifier that could well predict TGF- $\beta$ levels in different datasets of GIAD. In our study, TGF- $\beta^{\text {high }}$ patients displayed a high propensity of overexpressed mRNAs which are relevant to extracellular matrix organization and cellular biological process. These cell behaviors included proliferation, adhesion, invasion and migration, which can be inhibited by regulations at transcriptional level for TGF- $\beta^{\text {low }}$ patients. We also noticed that a number of metastasis promoting proteins were upregulated in TGF- $\beta^{\text {high }}$ samples with inhibiting proteins downregulated. In line with our finding, a previous research comprehensively identified the function of TGF- $\beta$ family in both transcriptional and molecular level [5]. Genomic analysis led to an impressed result that 
occurrence of significant alterations was tendentious across multiple cancer types (especially STAD). Specifically, a high level of TMB and SCNA were observed in TGF$\beta^{\text {low }}$ samples, which might produce more neoantigen that can be targeted at. Recently, immune responses were found to be suppressed due to TGF- $\beta$ induced abnormal function of immune cells within tumor microenvironment $[6,45,46]$. As for our observation, the immune resistance of TGF- $\beta$ signaling and poor genetic stability made the favorable prognosis explicable in TGF- $\beta^{\text {low }}$ patients.

Our analysis about drug sensitivity revealed that differentially altered gene were targeted by anticancer drugs for various clinical therapies. In particular, dabrafenib, accessible to a large number of oncogene targets, is one potential anticancer drug for GIAD patients. And lenalidomide might be a valid anticancer drug for TGF- $\beta^{\text {high }}$ patients. Though several trials of TGF- $\beta$ drugs have been implemented and reviewed, the molecular mechanisms of targeted drugs remained unspecific [7, 47-49]. The role of clinically actionable genes in cancer progression and metastasis deserves further explanation so that rational combination therapies can be exploitable to promote prognosis in cancer patients. Hence, our systematic identification of TGF- $\beta$ molecular signatures and sensitivity analysis can benefit clinical targeted therapies which are expected to be more efficient in the future.

This study has certain limitations. Despite these causalities and correlations elaborated by our and many previous studies, further experimentations and analyses can be helpful to extend these conclusions and clinical viability. Retrospective studies and large-scale clinical trials are necessary for experimental verification. Finally, our combination of multi-omics is comprehensive but not exhaustive. Potential regulatory pathways and molecular mechanisms need further investigation. In addition, prospective cohort studies are called to manifest clinical benefits for patients with tumor.

\section{Conclusions}

Our study provided a comprehensive analysis of the molecular characteristics associated with TGF- $\beta$ and provides possible therapeutic targets in GIAD. 


\section{Abbreviations}

GIAD, gastrointestinal adenocarcinoma; ESAD, esophageal adenocarcinoma; STAD, stomach adenocarcinoma; COAD, colon adenocarcinoma; READ, rectum adenocarcinoma; TGF- $\beta$, transforming growth factor beta; $\mathrm{CNV}$, copy number variation; ssGSEA, single sample gene-set enrichment analysis; DNN, deep neural network; EMT, epithelial mesenchymal transition; TMB, tumor mutation burden. OS, overall survival; PFI, progression-free interval; DFS, disease-free survival.

\section{Acknowledgements}

We are grateful to Master Huang Xin (Hunan University, college of electrical and information engineering) for his help in the schematic drawing of deep neural network.

\section{Authors' contributions}

BXZ and ZGZ conceived this study. BXZ, ZGZ and XPC provided financial and administrative support. QFZ and FRL did most of the data analysis. LQ, ZBL, and JS collected the public data. XPC provided the advices of this study. All authors discussed the results, explained them further and prepared the panels for these figures. QFZ and FRL wrote the manuscript. All authors read and approved the final manuscript.

\section{Funding}

This study was funded by the State Key Project on Infection Disease of China (No. 2018ZX10723204-003); the Chinese Ministry of Public Health for Key Clinical Project (No. [2010] 493-51); National Natural Science Foundation of China (81502530 and 81874189).

\section{Availability of data and materials}

Data in this study are from the TCGA, GDSC, and GEO database. To view additional 
421 sequencing data involved in this article please visit websites at:

422 https://www.ncbi.nlm.nih.gov/geo/query/acc.cgi?acc=GSE19417,

423 https://www.ncbi.nlm.nih.gov/geo/query/acc.cgi?acc=GSE62254,

424 https://www.ncbi.nlm.nih.gov/geo/query/acc.cgi?acc=GSE17536,

425 https://www.ncbi.nlm.nih.gov/geo/query/acc.cgi?acc=GSE45404,

426 https://www.ncbi.nlm.nih.gov/geo/query/acc.cgi?acc=GSE62254,

427 https://www.ncbi.nlm.nih.gov/geo/query/acc.cgi?acc=GSE17536. To view multi428 omics data of ESAD, STAD, COAD and READ please visit websites at:

429 https://xenabrowser.net/datapages/. Data downloading and processing are as described 430 in Methods and Materials. All data generated or analyzed during this study are included 431 in the manuscript and its additional files.

432

433 Ethics approval and consent to participate

434 Not applicable.

435 Consent for publication

$436 \quad$ Not applicable.

\section{Competing interests}

438 The authors declare that they have no competing interests.

439

440

\section{References}

1. Bray F, Ferlay J, Soerjomataram I, et al. Global cancer statistics 2018: GLOBOCAN estimates of incidence and mortality worldwide for 36 cancers in 185 countries. CA Cancer J Clin. 2018; 68:394-424.

445 2. Hoadley KA, Yau C, Hinoue T, et al. Cell-of-Origin Patterns Dominate the Molecular 
3. Liu Y, Sethi NS, Hinoue T, et al. Comparative Molecular Analysis of Gastrointestinal Adenocarcinomas. Cancer cell. 2018; 33:721-735.e728.

4. Zhang Y, Alexander PB, Wang XF. TGF-beta Family Signaling in the Control of Cell Proliferation and Survival. Cold Spring Harbor perspectives in biology. 2017; 9.

5. Morikawa M, Derynck R, Miyazono K. TGF-beta and the TGF-beta Family: Context-Dependent Roles in Cell and Tissue Physiology. Cold Spring Harbor perspectives in biology. 2016; 8.

6. Batlle E, Massague J. Transforming Growth Factor-beta Signaling in Immunity and Cancer. Immunity. 2019; 50:924-940.

7. Colak S, Ten Dijke P. Targeting TGF-beta Signaling in Cancer. Trends in cancer. 2017; 3:56-71.

8. Gu S, Zaidi S, Hassan MI, et al. Mutated CEACAMs Disrupt Transforming Growth Factor Beta Signaling and Alter the Intestinal Microbiome to Promote Colorectal Carcinogenesis. Gastroenterology. 2020; 158:238-252.

9. Blum AE, Venkitachalam S, Ravillah D, et al. Systems Biology Analyses Show Hyperactivation of Transforming Growth Factor-beta and JNK Signaling Pathways in Esophageal Cancer. Gastroenterology. 2019; 156:1761-1774.

10. Seoane J, Gomis RR. TGF-beta Family Signaling in Tumor Suppression and Cancer Progression. Cold Spring Harbor perspectives in biology. 2017; 9.

11. Monti S, Tamayo P, Mesirov J, et al. Consensus Clustering: A Resampling-Based Method for Class Discovery and Visualization of Gene Expression Microarray Data. Machine Learning. 2003; 52:91-118.

12. Liu F, Qin L, Liao Z, et al. Microenvironment characterization and multi-omics signatures related to prognosis and immunotherapy response of hepatocellular carcinoma. Experimental hematology \& oncology. 2020; 9:10.

13. Ye $\mathrm{Y}, \mathrm{Hu} \mathrm{Q}$, Chen $\mathrm{H}$, et al. Characterization of Hypoxia-associated Molecular Features to Aid Hypoxia-Targeted Therapy. Nature metabolism. 2019; 1:431-444.

14. Chou CH, Shrestha S, Yang CD, et al. miRTarBase update 2018: a resource for experimentally validated microRNA-target interactions. Nucleic acids research. 2018; 46:D296-d302.

15. Chen $\mathrm{Y}$, Wang $\mathrm{X}$. miRDB: an online database for prediction of functional microRNA targets. Nucleic acids research. 2020; 48:D127-d131.

16. Yu G, Wang LG, Han Y, et al. clusterProfiler: an R package for comparing biological themes among gene clusters. Omics : a journal of integrative biology. 2012; 16:284-287.

17. Iorio F, Knijnenburg TA, Vis DJ, et al. A Landscape of Pharmacogenomic Interactions in Cancer. Cell. 2016; 166:740-754.

18. Geeleher P, Zhang Z, Wang F, et al. Discovering novel pharmacogenomic biomarkers by imputing drug response in cancer patients from large genomics studies. Genome research. 2017; 27:1743-1751.

19. Liu F, Liao Z, Song J, et al. Genome-wide screening diagnostic biomarkers and the construction of prognostic model of hepatocellular carcinoma. Journal of cellular biochemistry. 2020; 121:2582-2594.

20. Qian W, Li N, Cao Q, et al. Thrombospondin-4 critically controls transforming growth factor beta1 induced hypertrophic scar formation. Journal of cellular physiology. 2018; 234:731-739.

21. Miao H, Wang N, Shi LX, et al. Overexpression of mircoRNA-137 inhibits cervical cancer cell invasion, migration and epithelial-mesenchymal transition by suppressing the TGF-beta/smad 
pathway via binding to GREM1. Cancer cell international. 2019; 19:147.

22. Yao Y, Zhou Z, Li L, et al. Activation of Slit2/Robo1 Signaling Promotes Tumor Metastasis in Colorectal Carcinoma through Activation of the TGF-beta/Smads Pathway. Cells. 2019; 8.

23. Chowdhury A, Herzog C, Hasselbach L, et al. Expression of fibulin- 6 in failing hearts and its role for cardiac fibroblast migration. Cardiovascular research. 2014; 103:509-520.

24. Hao Y, Baker D, Ten Dijke P. TGF-beta-Mediated Epithelial-Mesenchymal Transition and Cancer Metastasis. International journal of molecular sciences. 2019; 20.

25. David CJ, Huang YH, Chen M, et al. TGF- $\beta$ Tumor Suppression through a Lethal EMT. Cell. 2016; 164:1015-1030.

26. Zhao Y, Zhang F, Pan Z, et al. LncRNA NR_003923 promotes cell proliferation, migration, fibrosis, and autophagy via the miR-760/miR-215-3p/IL22RA1 axis in human Tenon's capsule fibroblasts. Cell death \& disease. 2019; 10:594.

27. Xu Z, Guo B, Chang P, et al. The Differential Expression of miRNAs and a Preliminary Study on the Mechanism of miR-194-3p in Keloids. BioMed research international. 2019; 2019:8214923.

28. Yi L, Ouyang L, Wang S, et al. Long noncoding RNA PTPRG-AS1 acts as a microRNA-194-3p sponge to regulate radiosensitivity and metastasis of nasopharyngeal carcinoma cells via PRC1. Journal of cellular physiology. 2019; 234:19088-19102.

29. Pan X, Zhao L, Quan J, et al. MiR-378a-5p acts as a tumor suppressor in renal cell carcinoma and is associated with the good prognosis of patients. American journal of translational research. 2019; 11:2207-2218.

30. Liu S, Yang Y, Jiang S, et al. MiR-378a-5p Regulates Proliferation and Migration in Vascular Smooth Muscle Cell by Targeting CDK1. Frontiers in genetics. 2019; 10:22.

31. Ottaviani S, Stebbing J, Frampton AE, et al. TGF-beta induces miR-100 and miR-125b but blocks let-7a through LIN28B controlling PDAC progression. Nature communications. 2018; 9:1845.

32. Lu M, Wu J, Hao ZW, et al. Basolateral CD147 induces hepatocyte polarity loss by E-cadherin ubiquitination and degradation in hepatocellular carcinoma progress. Hepatology (Baltimore, Md). 2018; 68:317-332.

33. Padmanaban $\mathrm{V}$, Krol I, Suhail $\mathrm{Y}$, et al. E-cadherin is required for metastasis in multiple models of breast cancer. Nature. 2019; 573:439-444.

34. Vaniotis G, Rayes RF, Qi S, et al. Collagen IV-conveyed signals can regulate chemokine production and promote liver metastasis. Oncogene. 2018; 37:3790-3805.

35. Kenny HA, Chiang CY, White EA, et al. Mesothelial cells promote early ovarian cancer metastasis through fibronectin secretion. The Journal of clinical investigation. 2014; 124:4614-4628.

36. Shah A, Melhuish TA, Fox TE, et al. TGIF transcription factors repress acetyl CoA metabolic gene expression and promote intestinal tumor growth. Genes \& development. 2019; 33:388-402.

37. Bykov VJN, Eriksson SE, Bianchi J, et al. Targeting mutant p53 for efficient cancer therapy. Nature reviews Cancer. 2018; 18:89-102.

38. Thorsson V, Gibbs DL, Brown SD, et al. The Immune Landscape of Cancer. Immunity. 2018; 48:812-830.e814.

39. Chakravarthy A, Khan L, Bensler NP, et al. TGF-beta-associated extracellular matrix genes link cancer-associated fibroblasts to immune evasion and immunotherapy failure. Nature communications. 2018; 9:4692.

40. Sooro MA, Zhang N, Zhang P. Targeting EGFR-mediated autophagy as a potential strategy for cancer therapy. International journal of cancer. 2018; 143:2116-2125. 
41. Yamaoka T, Ohba M, Ohmori T. Molecular-Targeted Therapies for Epidermal Growth Factor Receptor and Its Resistance Mechanisms. International journal of molecular sciences. 2017; 18.

42. Zhu XH, Wang JM, Yang SS, et al. Down-regulation of DAB2IP promotes colorectal cancer invasion and metastasis by translocating hnRNPK into nucleus to enhance the transcription of MMP2. International journal of cancer. 2017; 141:172-183.

43. Huaman J, Naidoo M, Zang X, et al. Fibronectin Regulation of Integrin B1 and SLUG in Circulating Tumor Cells. Cells. 2019; 8.

44. Pure E, Blomberg R. Pro-tumorigenic roles of fibroblast activation protein in cancer: back to the basics. Oncogene. 2018; 37:4343-4357.

45. Kanamori M, Nakatsukasa H, Okada M, et al. Induced Regulatory T Cells: Their Development, Stability, and Applications. Trends in immunology. 2016; 37:803-811.

46. Travis MA, Sheppard D. TGF-beta activation and function in immunity. Annual review of immunology. 2014; 32:51-82.

47. Wang $\mathrm{H}$, Chen $\mathrm{M}$, Sang $\mathrm{X}$, et al. Development of small molecule inhibitors targeting TGF-beta ligand and receptor: Structures, mechanism, preclinical studies and clinical usage. European journal of medicinal chemistry. 2020; 191:112154.

48. Lan Y, Zhang D, Xu C, et al. Enhanced preclinical antitumor activity of M7824, a bifunctional fusion protein simultaneously targeting PD-L1 and TGF-beta. Science translational medicine. 2018; 10.

49. Neuzillet C, Tijeras-Raballand A, Cohen R, et al. Targeting the TGFbeta pathway for cancer therapy. Pharmacology \& therapeutics. 2015; 147:22-31.

\section{Figure Legends}

Fig. 1 Unsupervised classification of TGF- $\beta$ status and prognosis in gastrointestinal adenocarcinoma (GIAD). a Heatmap based on cluster of 39 TGF- $\beta$ core genes. b Comparison of TGF- $\beta$ scores in two clusters across different tumors. c-h Kaplansurvival curves (including overall survival and progression-free interval) for different TGF- $\beta$ groups in GIAD. *: $\mathrm{P}<0.05, * *: \mathrm{P}<0.01, * * *: \mathrm{P}<0.001$.

Fig. 2 TGF- $\beta$ specific multi-omics signatures in GIAD. a Overview of analysis process in this study. $\mathbf{b}$ The relative abundance and the number of each molecular signature in TGF- $\beta^{\text {high }}$ and TGF- $\beta^{\text {low }}$ group. The dot plot represents the proportion of molecular characteristics of each tumor in all GIADs, and the bar plot represents the number of molecular signatures of each tumor in the TGF- $\beta^{\text {high }}$ and TGF- $\beta^{\text {low }}$ group $($ FDR $<0.05)$. 
Fig. 3 TGF- $\beta$ effects on mRNA and functional pathways. a The heatmap shows the most significantly upregulated 50 mRNAs in the TGF- $\beta^{\text {high }}$ group across each GIAD. b KEGG pathways enriched by significantly upregulated mRNA in the TGF- $\beta^{\text {high }}$ group (FDR < 0.05). c Overall gene-set enrichment analysis (GSEA) pathway enrichment analysis was performed for the differentially expressed mRNAs (TGF- $\beta^{\text {high }} /$ TGF- $\left.\beta^{\text {low }}\right)$.

Fig. 4 Comparison of miRNA, and protein expression in different TGF- $\beta$ status. a Correlation between the first principal component (PC1) of the expression of TGF- $\beta^{\text {low }}$ specific miRNAs and their predicted targeted TGF- $\beta^{\text {high }}$-specific mRNAs. b The heatmap shows the most significantly down-regulated 20 miRNAs in the TGF- $\beta^{\text {high }}$ group across each GIAD. c A network Schematic diagram of miR-215-3p, miR-378a$5 p$, and miR-194-3p and their predicted targeting TGF- $\beta^{\text {high }}$-specific mRNA in stomach adenocarcinoma. d Negative correlation between miR-194-3p expression and TGF- $\beta$ score in 4 types of GIAD (Pearson correlation). e The significantly differential proteins in TGF- $\beta^{\text {high }}$ and TGF- $\beta^{\text {low }}$ groups; Red indicates high protein expression in the TGF$\beta^{\text {high }}$ group, and blue indicates high protein expression in the TGF- $\beta^{\text {low }}$ group.

Fig. 5 Significant mutations and CNVs between the TGF- $\beta$ subgroups in GIAD. a-b Comparison of tumor mutation burden (TMB) and CNV burden in different TGF- $\beta$ cluster. $\mathbf{c}$ Mutation frequency of significant mutation gene in two TGF- $\beta$ subtypes in stomach adenocarcinoma (Fisher's exact test, FDR < 0.05). d Chromosome map shows significant amplification and deletion of chromosome regions in stomach adenocarcinoma (Fisher's exact test, FDR $<0.05$ ); The bar plot on the right panel shows the amplification of the chromosomal region where EGFR is located. *: $\mathrm{P}<0.05,{ }^{* *}$ : $\mathrm{P}$ $<0.01, * * *: \mathrm{P}<0.001$.

Fig. 6 Analysis of TGF- $\beta$ associated gene signatures on drug susceptibility in GIAD. a TGF- $\beta$ associated gene responses to drugs involved in different pathways; The red dots represent genes, the blue dots represent drugs, the green line indicates high gene expression is sensitive to drugs, and the pink line indicates high gene expression is 
599

600

601

602

603

604

605

606

607

608

609

610

611

612

613

614

615

616

617 Additional file 1.docx:

618

619

620

resistant to drugs. b Volcano plot showed imputed drug response differences in the TGF- $\beta$ subgroups in GIAD (most samples are ESAD samples); The blue dots indicate significant drug-sensitivity, and the red dots indicate significant drug-resistance (FDR $<0.05$ ). $\mathbf{c}$ The correlation between lenalidomide (or IPA-3) and TGF- $\beta$ score.

Fig. 7 Identification of two TGF- $\beta$ subtypes based on the deep neural network (DNN) associated with TGF- $\beta$ signatures. a Schematic diagram of a deep neural network for predicting TGF- $\beta$ subtypes. b The performance of our DNN classifier on training and testing sets; Left: area under roc curve (AUC) of receiver operating characteristic (ROC) curve across each tumor. Right: ROC curve of the DNN classifier in stomach adenocarcinoma. $\mathbf{c}$ TGF- $\beta$ score in different TGF- $\beta$ subtypes for each other dataset classified by the DNN model. d-g Kaplan-survival curves (including overall survival and disease-free survival) for different TGF- $\beta$ groups identified by DNN model in GSE62254 and GSE17536. h Gene-set enrichment analysis showed that TGF- $\beta$ and EMT pathways were significantly enriched in different TGF- $\beta$ groups identified by DNN model. *: $\mathrm{P}<0.05, * *: \mathrm{P}<0.01, * * *: \mathrm{P}<0.001$.

Title: Supplementary Figures

Description: Supplementary figures and figure legends for manuscript. 
Figures
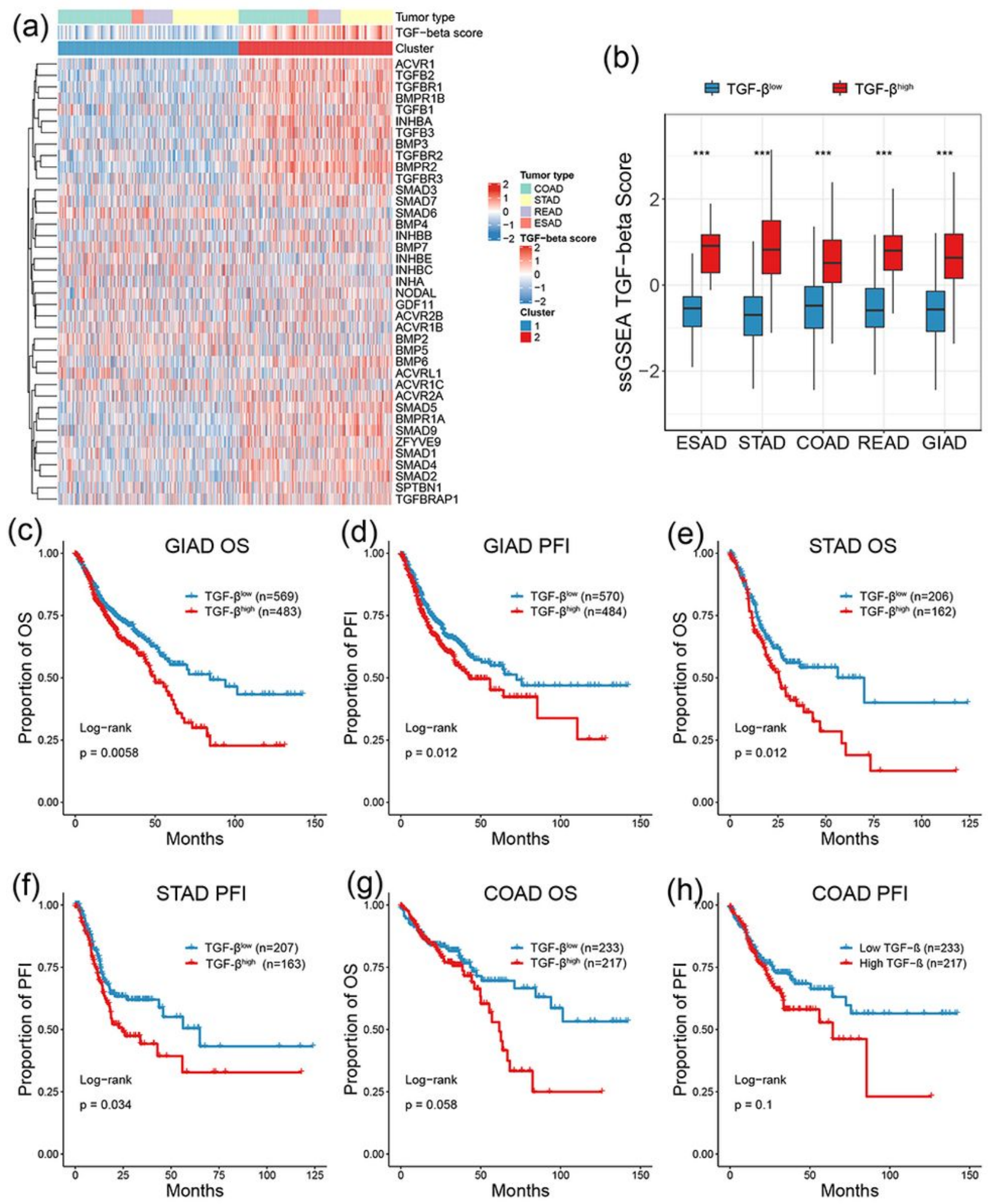

Figure 1

Unsupervised classification of TGF- $\beta$ status and prognosis in gastrointestinal adenocarcinoma (GIAD). a Heatmap based on cluster of 39 TGF- $\beta$ core genes. $b$ Comparison of TGF- $\beta$ scores in two clusters across 
different tumors. c-h Kaplan-survival curves (including overall survival and progression-free interval) for different TGF- $\beta$ groups in GIAD. *: $P<0.05, * *$ : $P<0.01$, ***: $P<0.001$.

(a)

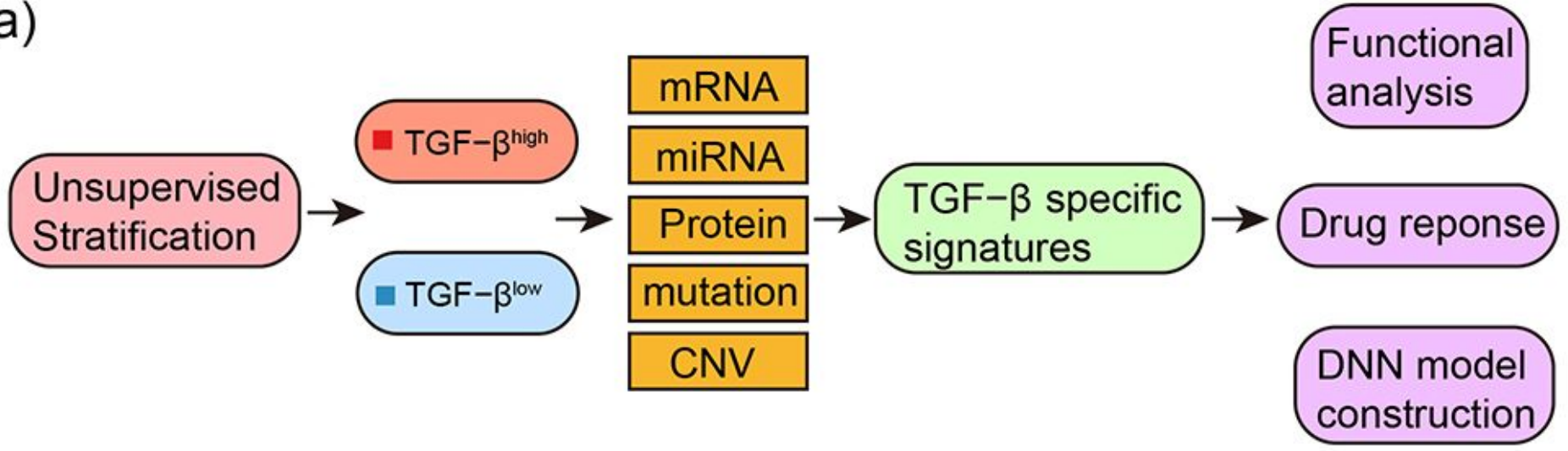

(b)

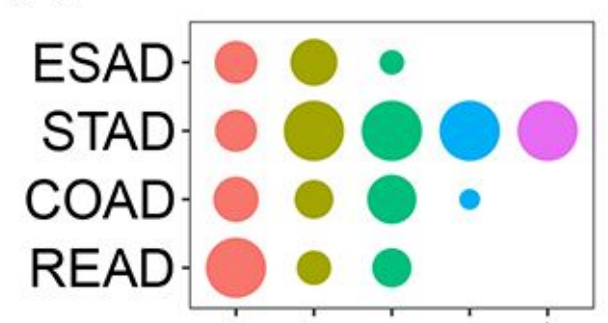

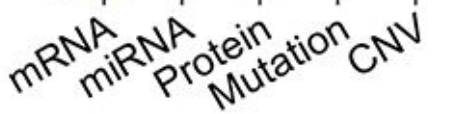

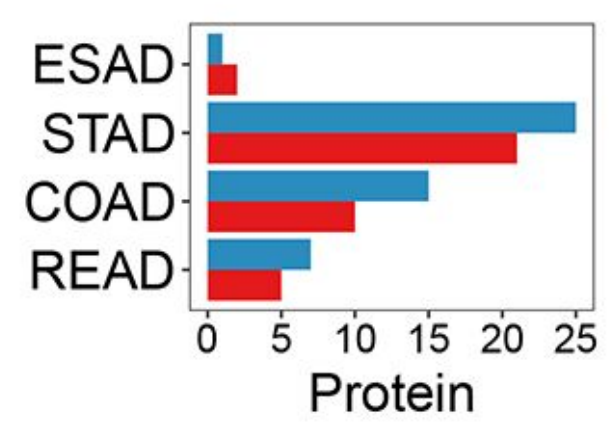

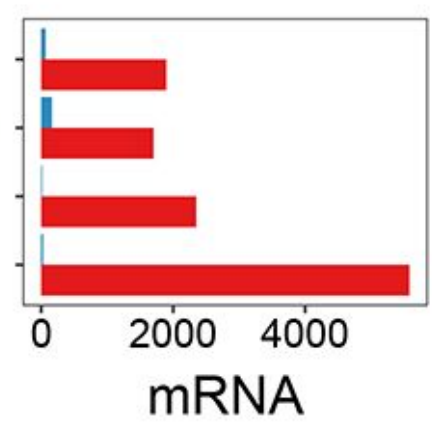

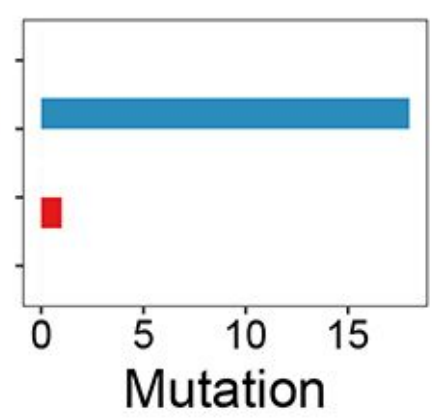

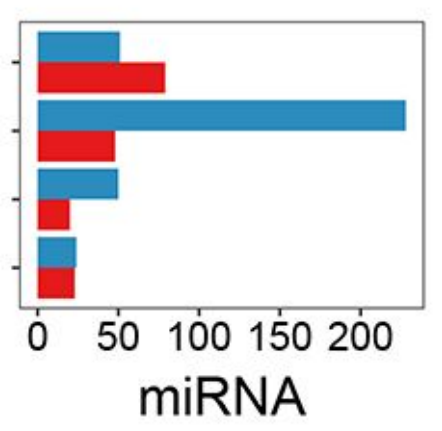

Normalized fraction
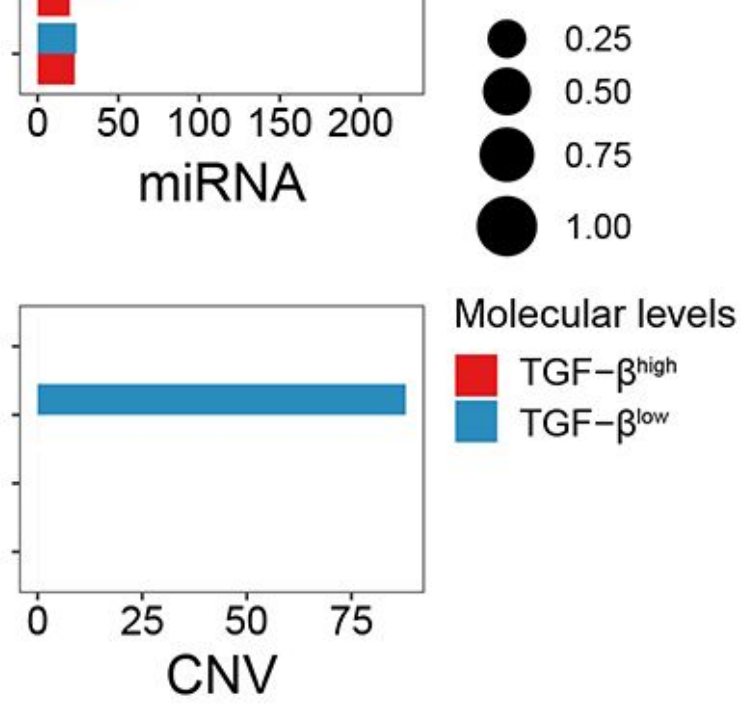

Figure 2

TGF- $\beta$ specific multi-omics signatures in GIAD. a Overview of analysis process in this study. $b$ The relative abundance and the number of each molecular signature in TGF- $\beta$ high and TGF- $\beta$ low group. The dot plot represents the proportion of molecular characteristics of each tumor in all GIADs, and the bar plot represents the number of molecular signatures of each tumor in the TGF- $\beta$ high and TGF- $\beta$ low group (FDR $<0.05)$. 
(a)

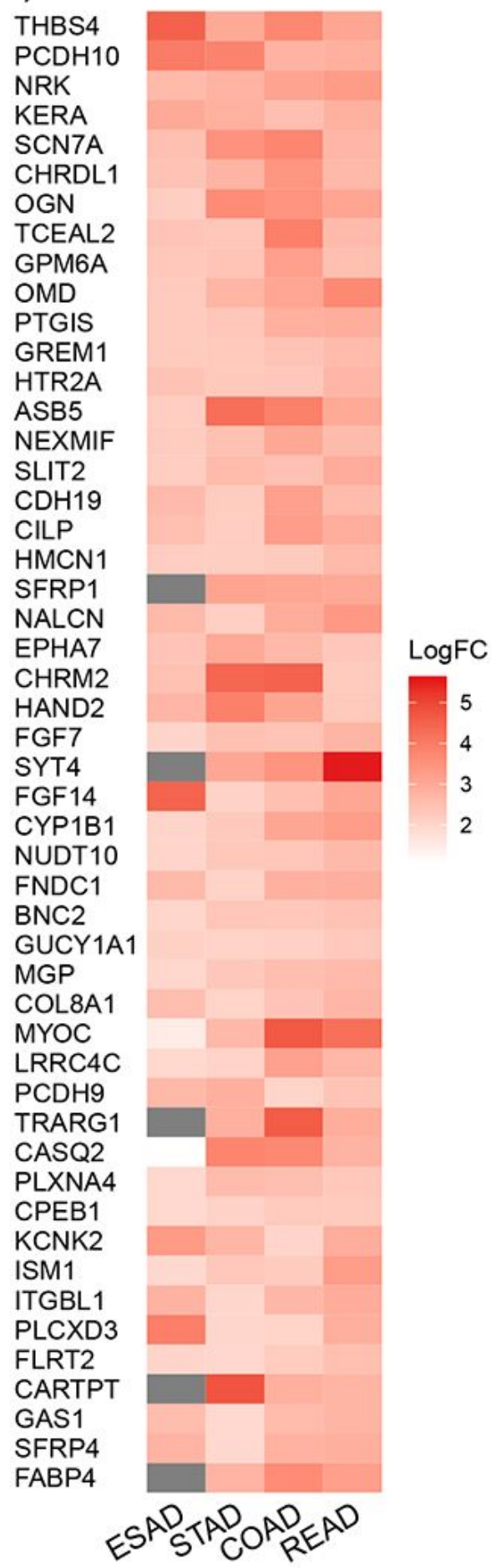

(b)

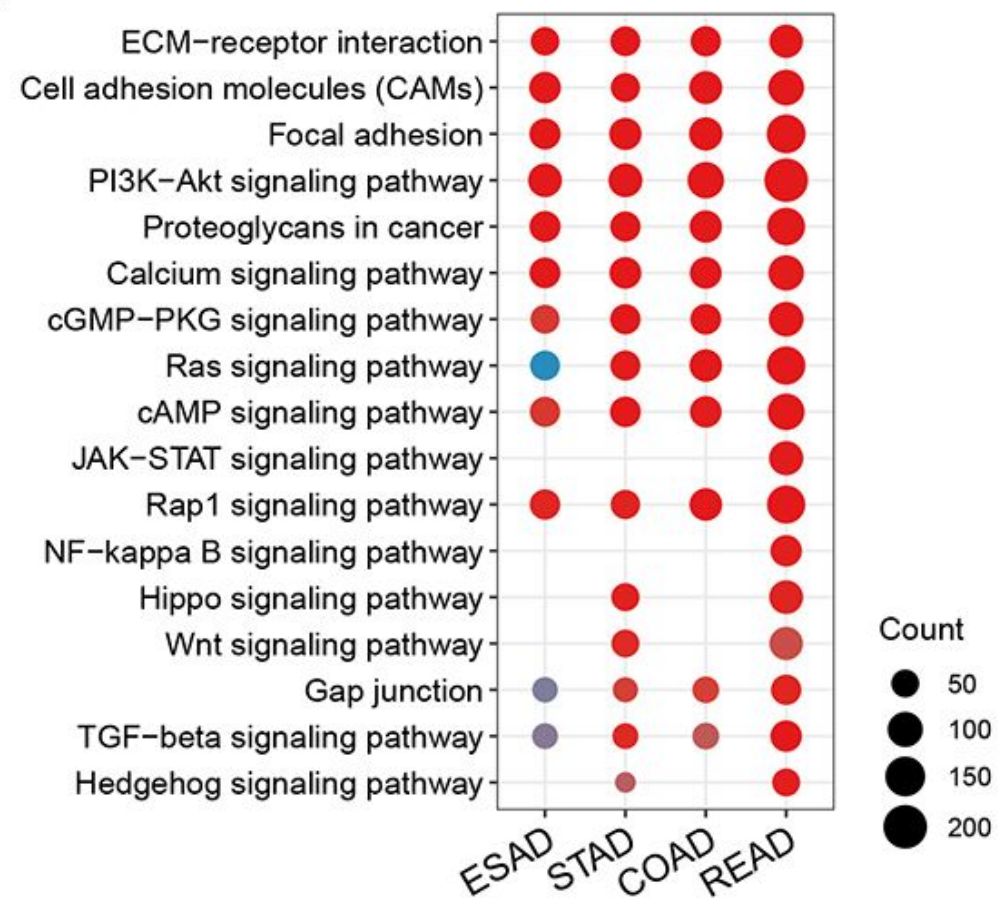

(c)

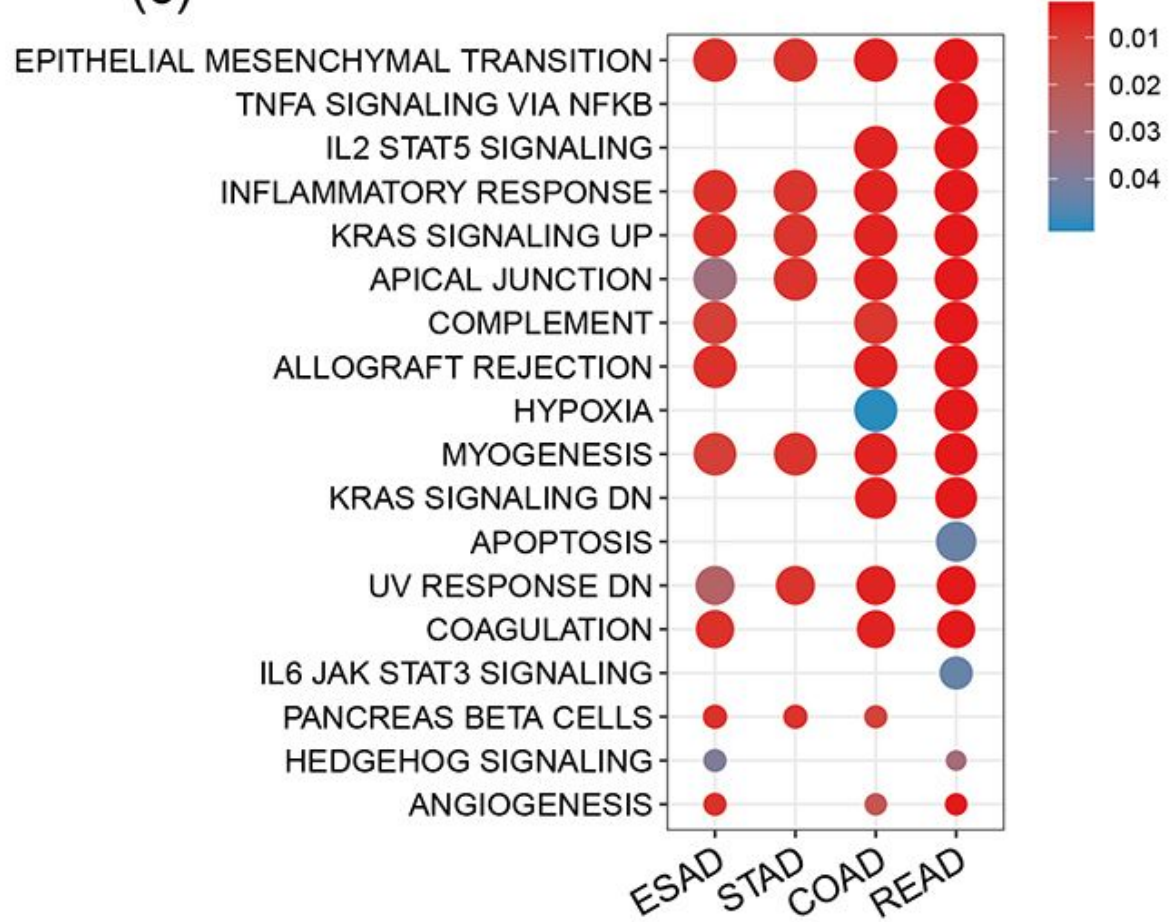

\section{Figure 3}

TGF- $\beta$ effects on mRNA and functional pathways. a The heatmap shows the most significantly upregulated 50 mRNAs in the TGF- $\beta$ high group across each GIAD. b KEGG pathways enriched by significantly upregulated mRNA in the TGF- $\beta$ high group (FDR < 0.05$)$. c Overall gene-set enrichment analysis (GSEA) pathway enrichment analysis was performed for the differentially expressed mRNAs

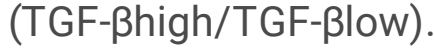


(a)

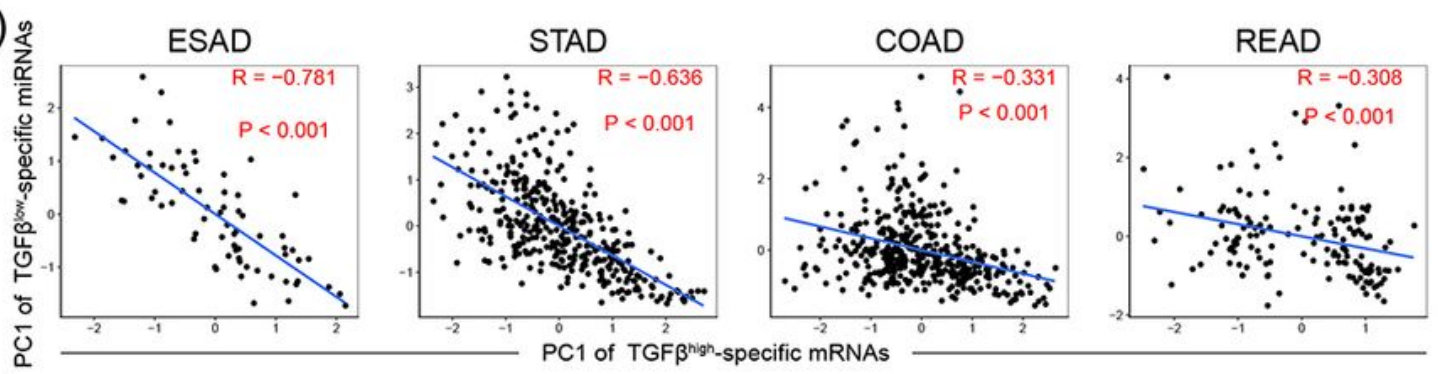

(b)

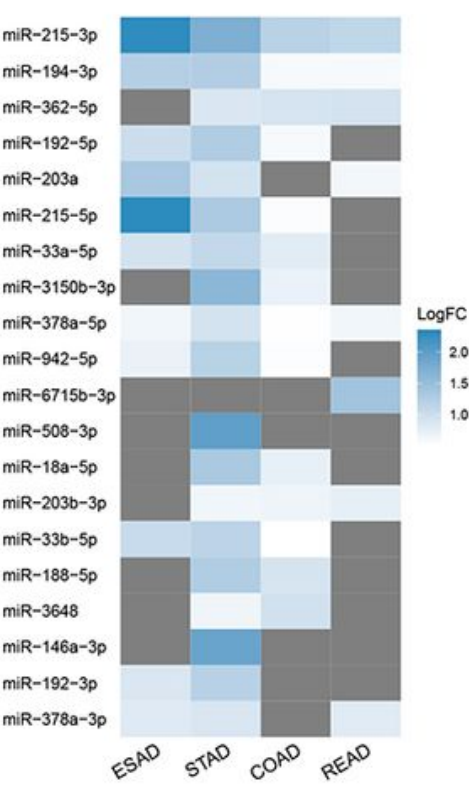

(d) (c)

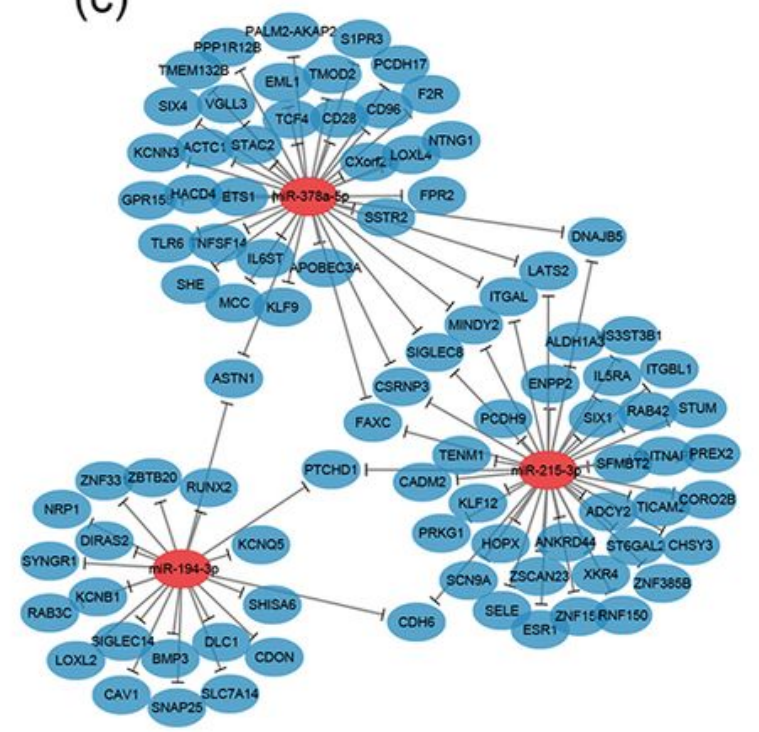

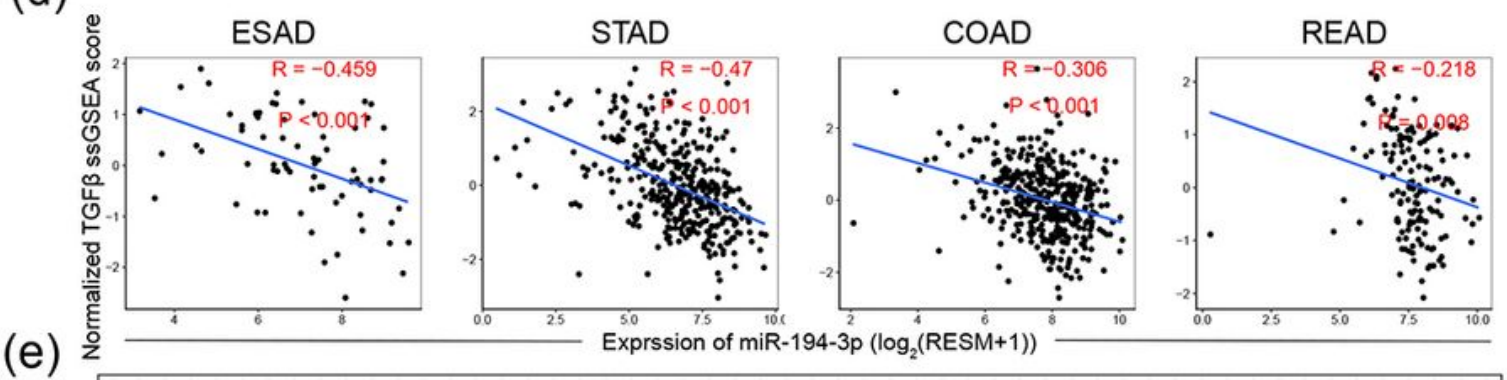

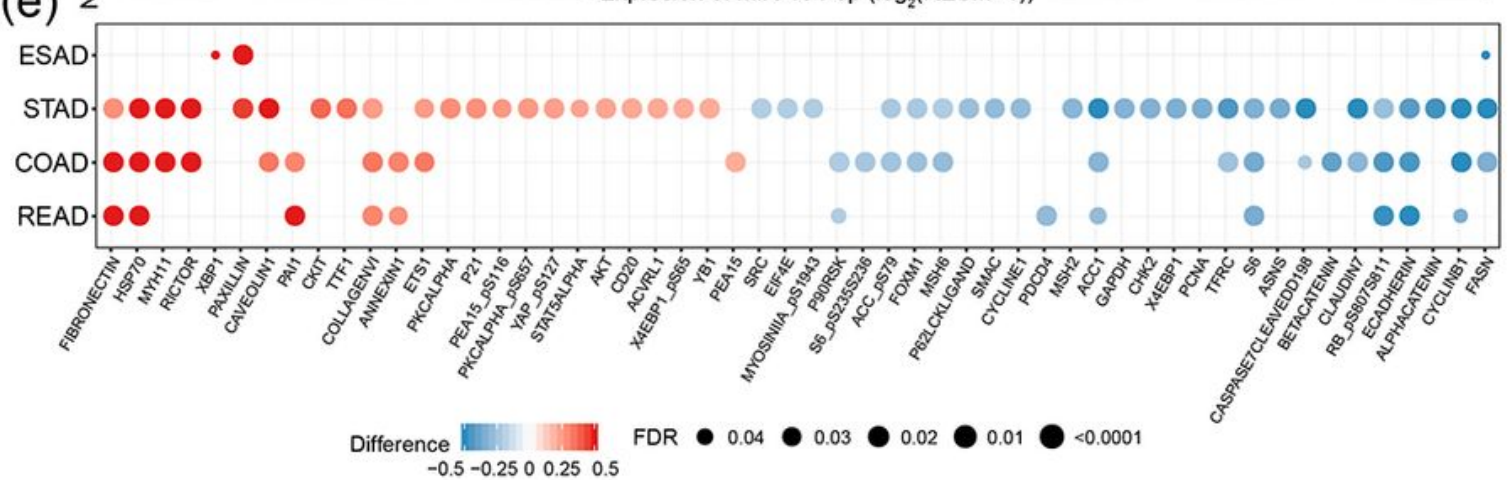

\section{Figure 4}

Comparison of miRNA, and protein expression in different TGF- $\beta$ status. a Correlation between the first principal component (PC1) of the expression of TGF- $\beta$ low-specific miRNAs and their predicted targeted TGF- $\beta$ high-specific mRNAs. b The heatmap shows the most significantly down-regulated 20 miRNAs in the TGF- $\beta$ high group across each GIAD. c A network Schematic diagram of miR-215-3p, miR-378a-5p, and miR-194-3p and their predicted targeting TGF- $\beta$ high-specific mRNA in stomach adenocarcinoma. $d$ 
Negative correlation between miR-194-3p expression and TGF- $\beta$ score in 4 types of GIAD (Pearson correlation). e The significantly differential proteins in TGF- $\beta$ high and TGF- $\beta$ low groups; Red indicates high protein expression in the TGF- $\beta$ high group, and blue indicates high protein expression in the TGFBlow group.
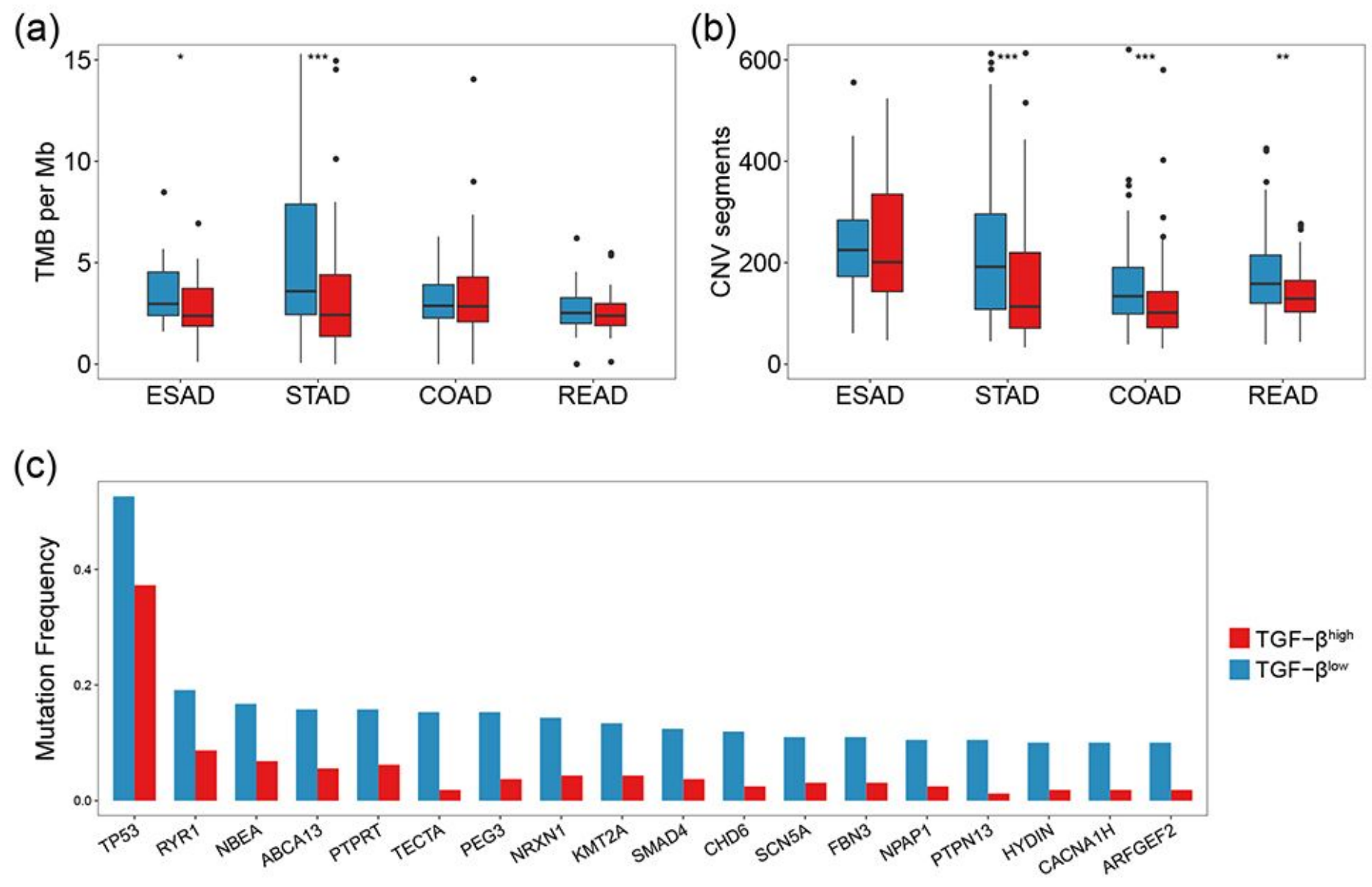

(d)
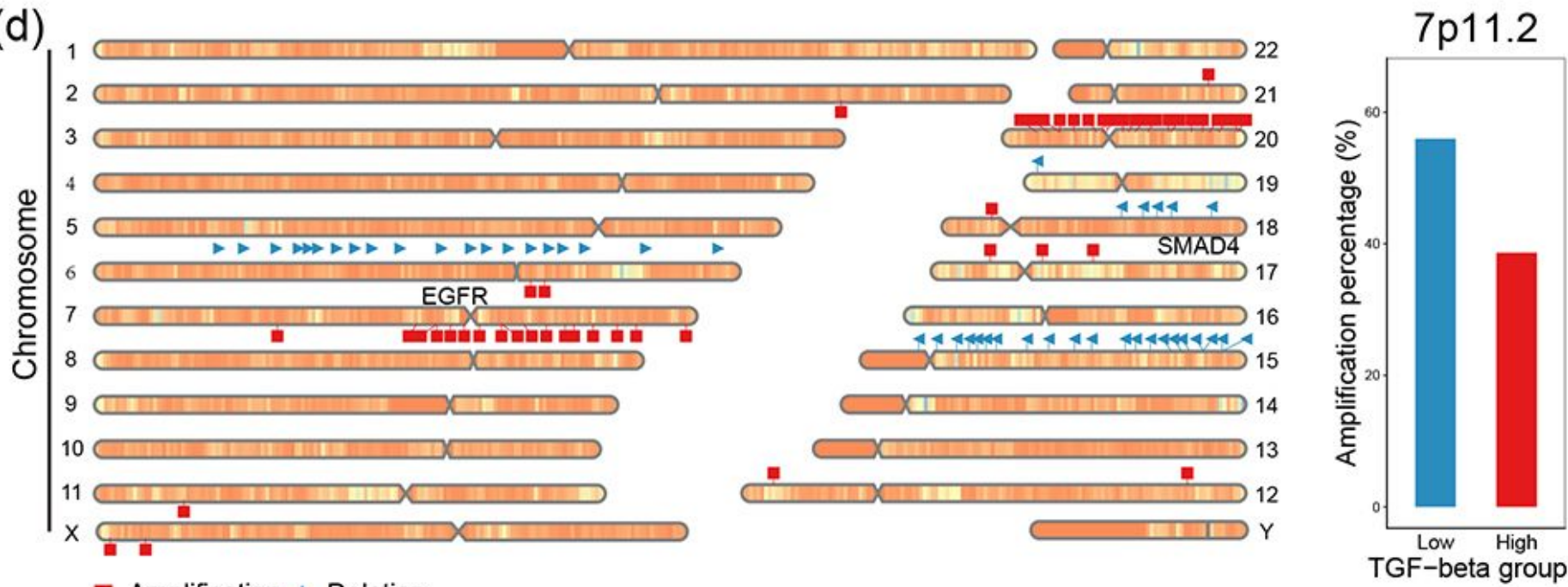

Figure 5 
Significant mutations and CNVs between the TGF- $\beta$ subgroups in GIAD. a-b Comparison of tumor mutation burden (TMB) and CNV burden in different TGF- $\beta$ cluster. c Mutation frequency of significant mutation gene in two TGF- $\beta$ subtypes in stomach adenocarcinoma (Fisher's exact test, FDR $<0.05$ ). $d$ Chromosome map shows significant amplification and deletion of chromosome regions in stomach adenocarcinoma (Fisher's exact test, FDR $<0.05$ ); The bar plot on the right panel shows the amplification of the chromosomal region where EGFR is located. *: $\mathrm{P}<0.05, * *: \mathrm{P}<0.01$, ***: $\mathrm{P}<0.001$.

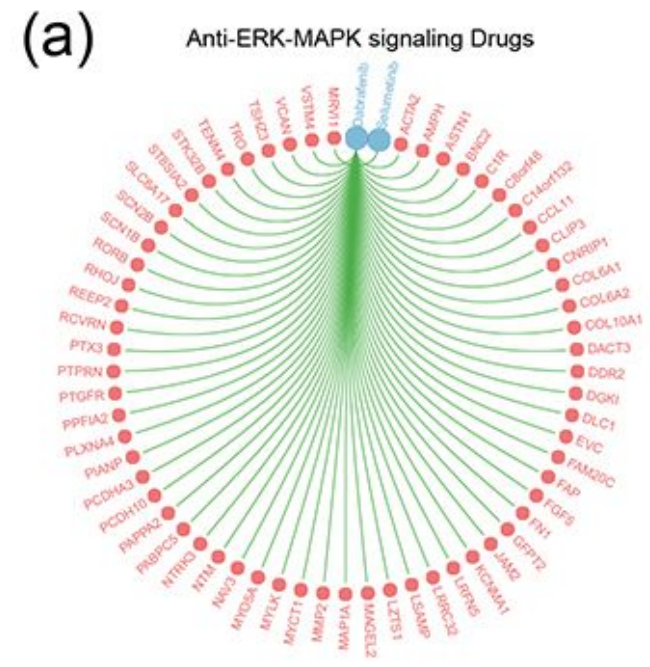

(b)

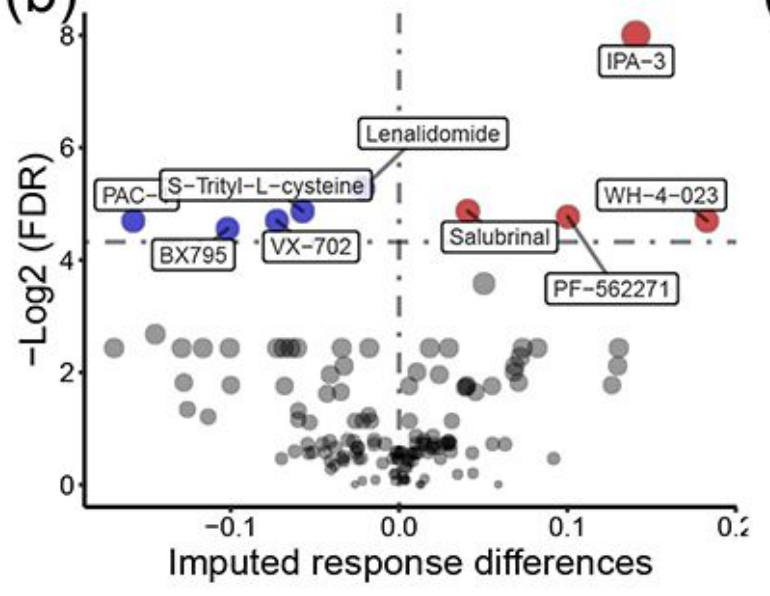

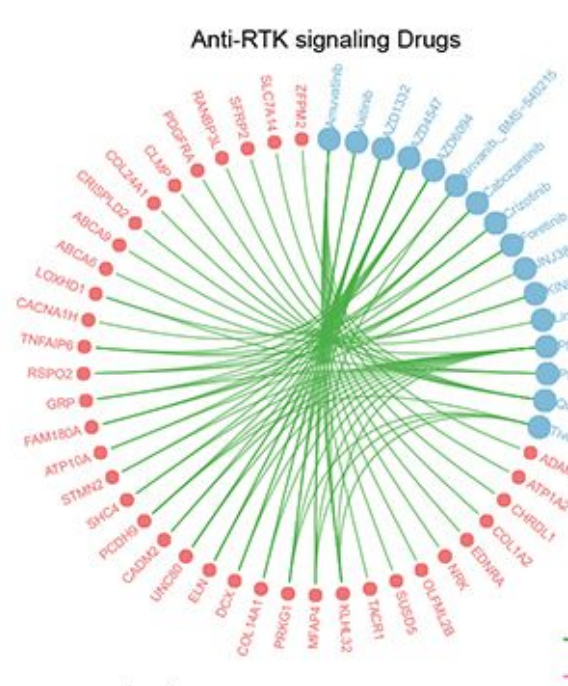

(c)

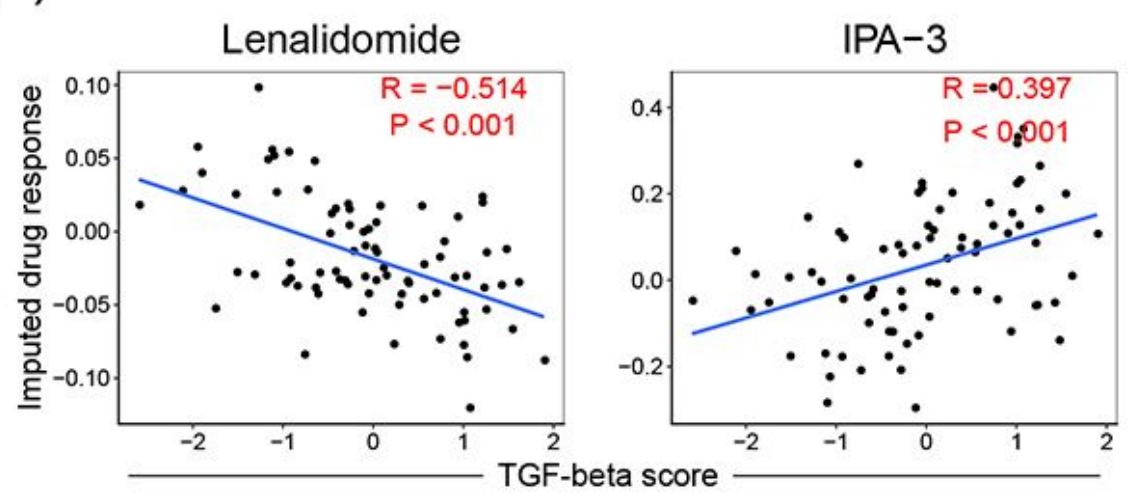

Figure 6

Analysis of TGF- $\beta$ associated gene signatures on drug susceptibility in GIAD. a TGF- $\beta$ associated gene responses to drugs involved in different pathways; The red dots represent genes, the blue dots represent drugs, the green line indicates high gene expression is sensitive to drugs, and the pink line indicates high gene expression is resistant to drugs. $b$ Volcano plot showed imputed drug response differences in the TGF- $\beta$ subgroups in GIAD (most samples are ESAD samples); The blue dots indicate significant drugsensitivity, and the red dots indicate significant drug-resistance (FDR $<0.05)$. c The correlation between lenalidomide (or IPA-3) and TGF- $\beta$ score. 
(a) gene

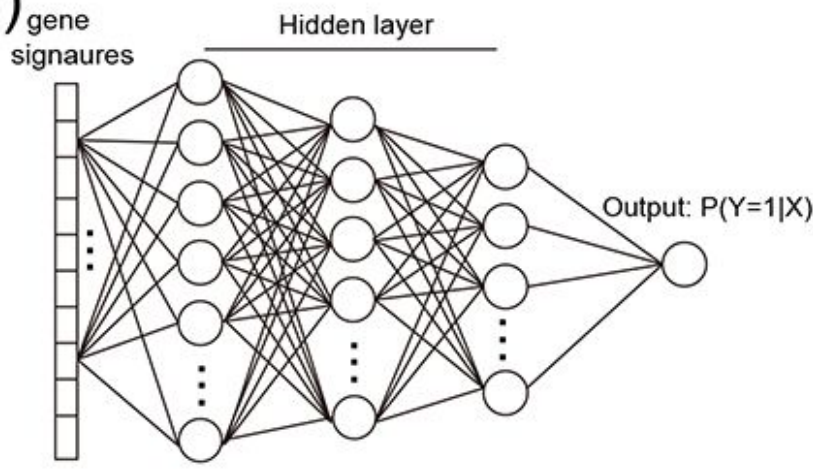

(c)
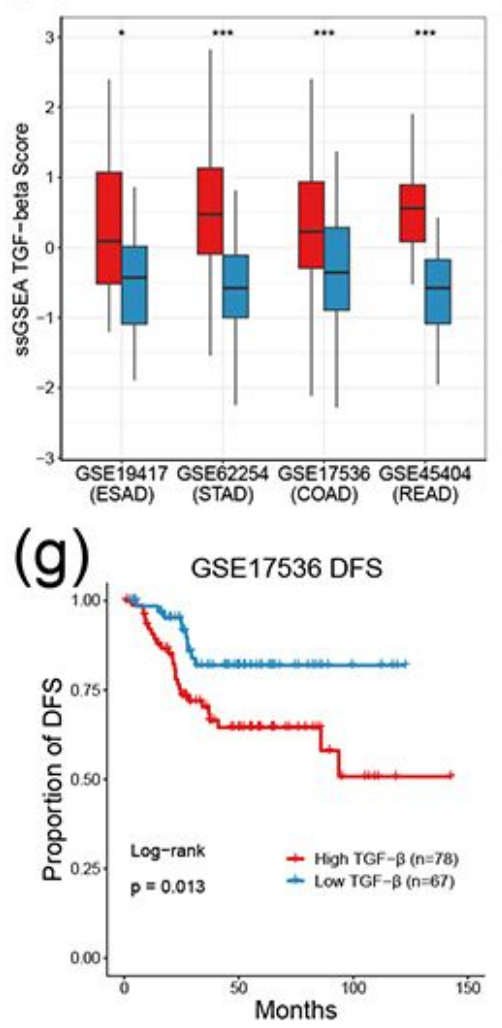

(b)

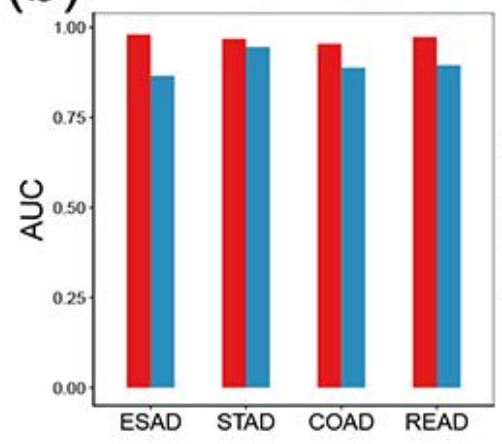

(e)
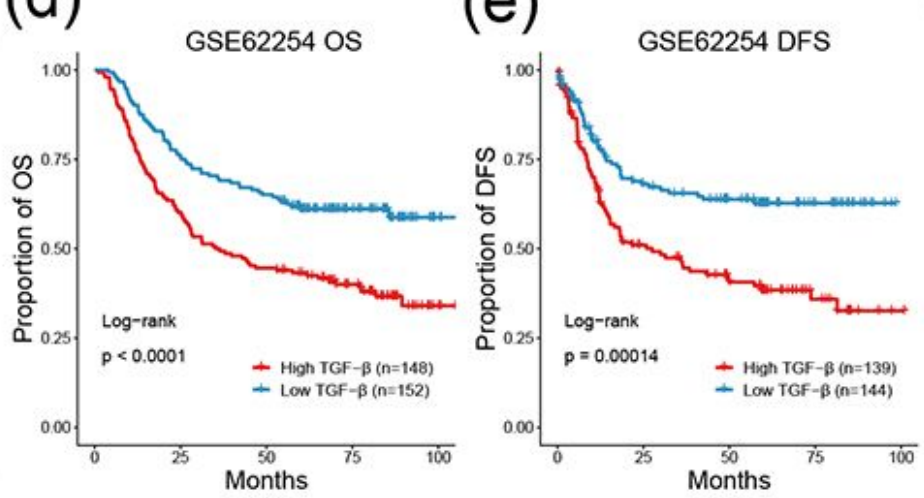

STAD model

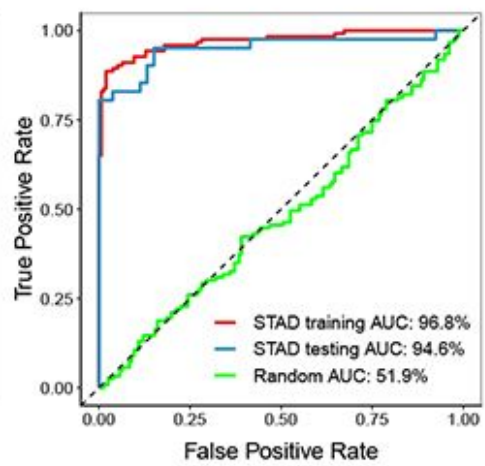

(f)

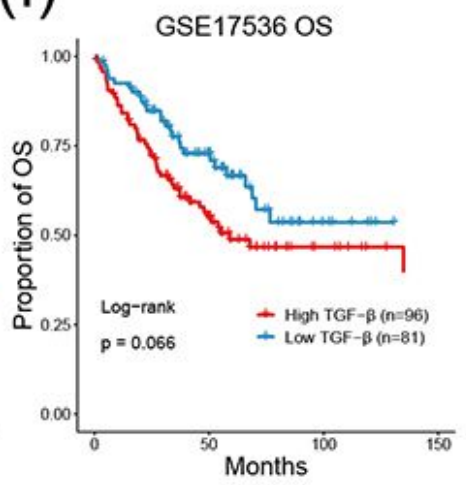

(h)

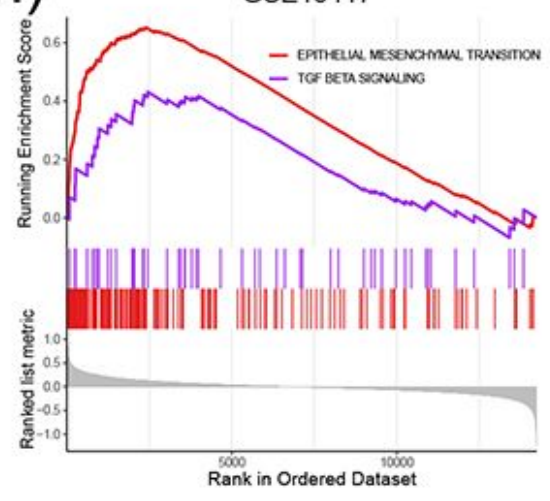

GSE62254

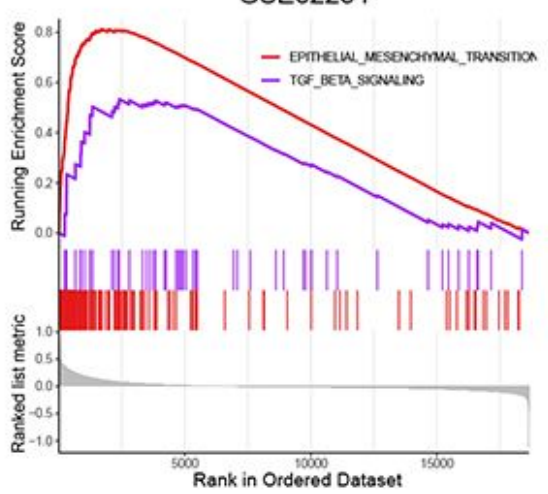

Figure 7

Identification of two TGF- $\beta$ subtypes based on the deep neural network (DNN) associated with TGF- $\beta$ signatures. a Schematic diagram of a deep neural network for predicting TGF- $\beta$ subtypes. b The performance of our DNN classifier on training and testing sets; Left: area under roc curve (AUC) of receiver operating characteristic (ROC) curve across each tumor. Right: ROC curve of the DNN classifier in stomach adenocarcinoma. c TGF- $\beta$ score in different TGF- $\beta$ subtypes for each other dataset classified by the DNN model. d-g Kaplan-survival curves (including overall survival and disease-free survival) for different TGF- $\beta$ groups identified by DNN model in GSE62254 and GSE17536. h Gene-set enrichment analysis showed that TGF- $\beta$ and EMT pathways were significantly enriched in different TGF- $\beta$ groups identified by DNN model. *: $P<0.05$, **: $P<0.01$, ***: $P<0.001$. 


\section{Supplementary Files}

This is a list of supplementary files associated with this preprint. Click to download.

- Additionalfile1.docx 